\title{
SoccerCraft: Relato de Atividade para Ensino Aprendizagem de Habilidades do Pensamento Computacional Aplicada no Sexto Ano do Ensino Fundamental ${ }^{*}$
}

\author{
Simão Martin ${ }^{1}$, Simone Cavalheiro ${ }^{1}$, Renata Reiser $^{1}$, \\ Luciana Foss $^{1}$, Ana Rita Mazzini ${ }^{1}$, André Du Bois ${ }^{1}$, \\ Clause Piana ${ }^{1}$ \\ ${ }^{1}$ Centro de Desenvolvimento Tecnológico - Universidade Federal de Pelotas (UFPel), \\ Rua Gomes Carneiro, 1 - 96.010-610 - Pelotas - RS, Brasil
}

\begin{abstract}
Computational Thinking (CT) is a fundamental skill in many areas and involves several techniques for solving problems, based on fundamental concepts of Computer Science. This paper presents an activity proposal for the sixth year of elementary school that develops algorithmic thinking, one of the abilities of the CT. The methodology consists of playful and fun tasks, without the use of the computer, that simulate a soccer match with characters similar to those of the Minecraft game. The activity is denominated SoccerCraft. In addition to the proposed methodology, the results and reports of the application of the activity to a class of a public school in the city of Pelotas are also presented.
\end{abstract}

\begin{abstract}
Resumo. O Pensamento Computacional (PC) é uma habilidade fundamental para diversas áreas e envolve várias técnicas para a resolução de problemas, embasadas em conceitos fundamentais da Ciência da Computação. Este artigo apresenta uma proposta de atividade para o sexto ano do ensino fundamental que desenvolve o pensamento algorítmico, uma das habilidades do PC. A metodologia consiste em tarefas lúdicas e divertidas, sem o uso do computador, que simulam um jogo de futebol com personagens similares aos do jogo Minecraft. Esta atividade foi denominada SoccerCraft. Além da metodologia proposta, também são apresentados os resultados e relatos da aplicação da atividade a uma turma de uma escola pública do município de Pelotas.
\end{abstract}

\section{Introdução}

O Pensamento Computacional (PC, do inglês Computational Thinking) consiste em uma metodologia para resolução de problemas baseada nos conceitos da ciência da computação [Wing 2006]. O PC é um método fundamental para todos que utiliza conceitos e técnicas aplicados na criação de programas computacionais, com o intuito de resolver problemas de diversas áreas, não necessariamente ligadas à computação [Marques et al. 2017, Barbosa et al. 2017, dos Reis et al. 2018].

Neste contexto, torna-se relevante estimular as crianças a terem contato desde cedo com o PC [França et al. 2012]. Com o propósito de auxiliar esse precoce incremento e consolidação do raciocínio lógico e computacional nas crianças, as escolas de Ensino Fundamental são consideradas locais importantes para a introdução desta

*Projeto realizado com o apoio da PREC, PRPPG e PPGC / UFPel 
metodologia. Vários trabalhos têm sido desenvolvidos neste sentido, tanto nacionalmente, [Barbosa et al. 2017, França et al. 2012, Santos et al. 2016], quanto internacionalmente, como a proposta da Google, em 2013, apresentando um conjunto de atividades do PC no intuito de auxiliar alunos do ensino primário e secundário dos Estados Unidos [Google 2013].

Por sua vez, o futebol destaca-se como o esporte mais praticado e difundido entre as crianças brasileiras e é adorado pela maioria delas. Um dos jogos eletrônicos que atualmente faz sucesso dentre as crianças é o jogo Minecraft [Persson and Bergensten 2011], sendo o terceiro mais jogado do mundo [TNH1 2017].

Motivados por este contexto, este trabalho apresenta a concepção, modelagem e desenvolvimento de um jogo educacional denominado SoccerCraft, considerando também uma etapa de validação, na qual são descritos e relatados o conjunto de atividades aplicadas em alunos de escola pública. A metodologia proposta explora regras de futebol utilizando personagens similares aos do jogo Minecraft com o intuito de desenvolver, via estratégias inerentes ao PC, a habilidade de pensamento algorítmico nos alunos do sexto ano do ensino fundamental. Além do incentivo ao desenvolvimento do raciocínio lógico via PC, dentre os objetivos secundários que podem ser alcançados pode-se citar o incremento da motivação do aluno em participar das aulas e das atividades propostas.

Este artigo está estruturado com a seguinte organização: a Seção 2 apresenta uma breve explicação sobre o conceito de PC e suas particularidades. A Seção 3 descreve a proposta metodológica utilizada na aplicação da atividade, incluindo materiais utilizados e os planos de aula. Na sequência, segue a Seção 4 que relata a aplicação da atividade, além de apresentar e discutir os resultados provenientes das tarefas. Na conclusão, têm-se as considerações finais e continuidade do trabalho.

\section{Pensamento Computacional}

Segundo a Sociedade Internacional de Tecnologia na Educacão (ISTE), em [ISTE 2013], o PC é um processo de resolucão de problemas que inclui (mas não é limitado a) as seguintes características:

- Reformular um problema no intuito de resolvê-lo mais facilmente.

- Organizar dados logicamente.

- Analisar dados complexos por partes, utilizando abstração e decomposição.

- Utilizar algoritmos para a automatização de soluções complexas.

- Formular soluções mais eficientes para problemas já resolvidos.

- Generalizar a solução de um problema para a resolução de problemas mais amplos.

A ISTE, juntamente com a CSTA (Computer Science Teachers Association) e a NSF (National Science Foundation), elaboraram um conjunto de ferramentas visando a competência de habilidades básicas do PC no Ensino Médio chamado Computational Thinking in K-12 Education Leadership Toolkit. As ferramentas apresentadas nesse toolkit apresentam nove conceitos fundamentais do PC em um quadro de progressão, são eles: coleta de dados, análise de dados, representação de dados, decomposição de problemas, algoritmos e procedimentos, automação, simulação e paralelismo. 
Existe uma ampla gama de projetos com o objetivo de levar à escolas do Ensino Básico atividades para a difusão do PC em diversos níveis de ensino. Alguns trabalhos relacionados estrangeiros merecem destaque, como [Barr and Stephenson 2011, Carnegie Mellon 2013, Google 2013, Lee et al. 2011, Kafura and Tatar 2011, Lugo and Olabe 2018].

No Brasil existem alguns projetos do PC que merecem destaque como, por exemplo, o de [Teixeira et al. 2015] que apresenta o projeto entitulado "Escola de Hackers", no município de Passo Fundo-RS, no qual mais de 300 alunos do $6^{\circ}$ ao $9^{\circ}$ ano do Ensino Fundamental, entre 11 e 14 anos, utilizam a ferramenta Scratch para desenvolver competências na área de programação de computadores e de raciocínio lógico matemático. Nacionalmente também se destaca o Computação Desplugada, que tem o objetivo de divulgar fundamentos da Ciência da Computação sem o uso do computador para alunos de escolas públicas [Silva et al. 2017] e o Explorando o PC para a Qualificação do Ensino Fundamental (ExpPC) [UFPel 2018], que visa a elaboração e aplicação de atividades lúdicas e interessantes que desenvolvam conceitos considerados essenciais no PC, para estudantes de escolas da rede pública [Campos et al. 2014, Junior et al. 2017, Marques et al. 2017, Weisshahn et al. 2016]. No Brasil ainda há trabalhos como os de [França et al. 2012, Zanetti et al. 2017, Batista et al. 2015].

\section{Metodologia da Atividade}

Este trabalho, que foi concebido junto ao projeto ExpPC, tem por objetivo trabalhar os conceitos básicos de algoritmos mediante a aplicação do jogo educacional denominado SoccerCraft. Sua proposta de metodologia defende utilizar uma abordagem de aprendizagem de algoritmos baseada em um jogo de futebol com personagens semelhantes aos do jogo Minecraft, sendo esse o principal diferencial deste trabalho em relação às demais abordagens da literatura. A atividade proposta foi dividida em cinco aulas, cada uma sendo um pouco mais complexa que a anterior.

O professor introduz novos conceitos de algoritmos à medida que as regras do jogo são adicionadas ao decorrer das aulas. As tarefas não possuem dependência de computadores. O público-alvo foram crianças do sexto ano do Ensino Fundamental de uma escola da rede pública de Pelotas, no RS. Essas aulas, seus materiais e seus conteúdos estão descritos no decorrer deste capítulo.

A atividade foi aplicada majoritariamente em dois encontros semanais, em aulas de 50 minutos, organizada em cinco tarefas, abordando conceitos e exercícios ligados a algoritmos. O detalhamento do material, planos desenvolvidos, fotos e instruções, podem ser obtidos no site ${ }^{1}$ do projeto. Com o intuito de medir a aprendizagem dos alunos quanto às habilidades em algoritmos, elaborou-se um total de 4 testes, os três primeiros aplicados nas três primeiras aulas e um teste final aplicado na quinta e última aula, este envolvendo todos os conceitos abordados.

\subsection{Tarefa I: Introdução ao SoccerCraft e Exercícios Iniciais}

Objetivos: esta tarefa tem como finalidade oferecer uma introdução aos alunos de como é o jogo SoccerCraft em seu modo básico, apresentar alguns exemplos de execução do

\footnotetext{
${ }^{1}$ https://wp.ufpel.edu.br/pensamentocomputacional/pt/
} 


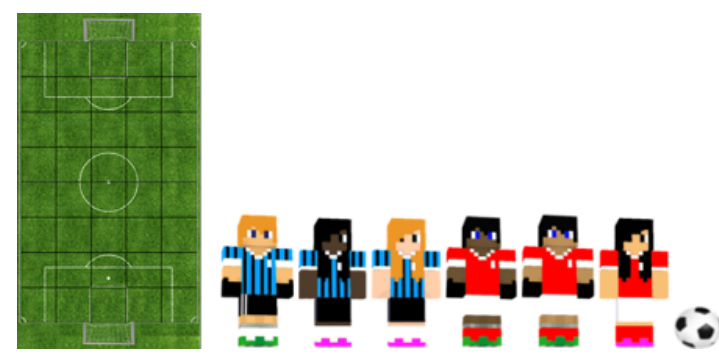

Figura 1. Campo, jogadores e bola do jogo SoccerCraft.

jogo e realizar alguns exercícios simplificados. Aqui é introduzido comandos básicos e o conceito de sequência, despertando nos alunos o pensamento algorítmico, uma das habilidades do PC.

Material: a realização dessa tarefa utiliza alguns materiais, são eles:

- Campo de futebol fabricado em isopor ou algum material de fácil fixação de papéis com alfinetes. O campo deve ter linhas horizontais e verticais dividindo-o em quadrados. A Figura 1 exemplifica esse campo.

- Uma bola e personagens estilo Minecraft [Persson and Bergensten 2011] impressos em cartolina ou em algum material mais resistente que papel, apresentados também na Figura 1. Os jogadores preferencialmente devem ser de várias etnias e ambos sexos, a fim de facilitar a imersão dos alunos dentro do jogo. Os uniformes dos jogadores podem variar de acordo com as cores dos principais times da região para uma maior proximidade do aluno.

- Alfinetes para fixação dos jogadores e bola no campo.

- Folhas de exercícios utilizando metade do campo de jogo, cada um com um cenário diferente (posicionamento diferente dos jogadores), com o objetivo de criar um algoritmo para fazer o gol com o menor número de unidades de tempo possível.

Metodologia: inicialmente são mostrados os materiais utilizados no jogo bem como explicadas as regras do mesmo, familiarizando o aluno ao SoccerCraft. As quatro regras iniciais são: (i) só o jogador que está com a bola pode se movimentar; (ii) o jogador só pode se movimentar por exatamente um quadrado; (iii) não podem haver dois jogadores no mesmo quadrado; e (iv) um jogador somente pode fazer gol de dentro da pequena área, sendo que cada movimento conta 1 minuto (cronômetro). Nesta fase, além do jogador com a bola, há somente adversários no campo. Os alunos devem escrever um algoritmo objetivando fazer o gol com apenas 4 comandos (movimentos) possíveis, que são: correr para direita, correr para esquerda, correr para frente e chutar. Caso não haja nenhum adversário no caminho e no momento do chute o jogador estiver dentro da pequena área, ele faz o gol. Considerando o cenário de jogo da Figura 2, um exemplo de algoritmo que permite fazer gol a partir do jogador azul com a bola, seria descrita como segue: correr para direita, correr para frente, correr para frente, correr para frente, correr para esquerda, chutar. Após a explicação do jogo utilizando o material, é entregue aos alunos uma folha de exercícios contendo novos cenários para que criem algoritmos visando fazer gol usando somente os comandos (movimentos) aprendidos até esta etapa. No final da aula, os exercícios são recolhidos para posterior correção. 


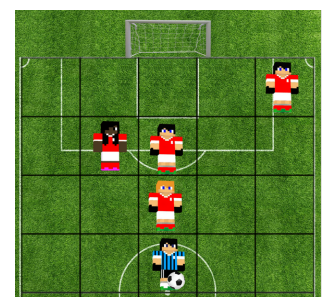

Figura 2. Exemplo de campo inicial do jogo SoccerCraft.

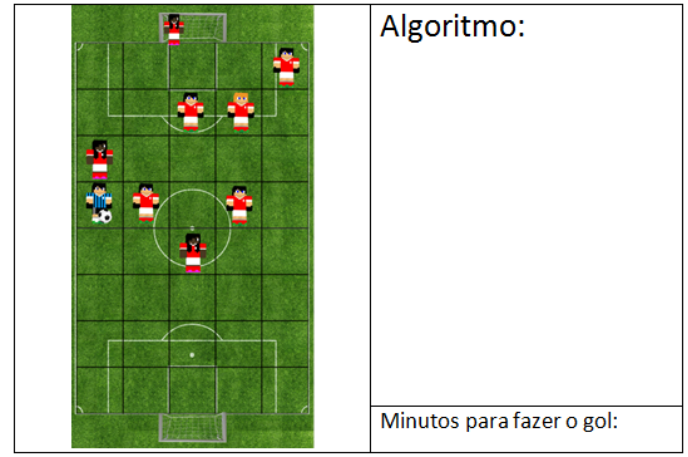

Figura 3. Exemplo de questão utilizada nos exercícios (testes) da aula 2.

\subsection{Tarefa II: Incrementando o nível de Dificuldade do Jogo e a Adição do Goleiro}

Objetivos: esta tarefa tem como finalidade incrementar a dificuldade do jogo SoccerCraft em relação à última aula, aumentando o tamanho do campo, adicionando o conceito do goleiro e adicionando quatro novos comandos. As habilidades do PC exploradas nesta tarefa são o pensamento algorítmico e a análise de custos.

Material: mesmos materiais da aula anterior (campo, jogadores e bola), um goleiro feito do mesmo material dos outros jogadores e também exercícios utilizando o campo completo de jogo, com diferentes cenários. A Figura 3 mostra um exemplo de exercício.

Metodologia: a atividade é iniciada relembrando aos alunos como jogar o SoccerCraft e revisando as estratégias consolidadas na última aula. O passo seguinte é a explicação de um novo comando, o "correr para trás". Agora o jogador com a bola também pode andar um quadrado para trás com o mesmo custo dos outros comandos (uma unidade de tempo). Em seguida insere-se o conceito do goleiro, localizado embaixo das traves ocupando as posições: central, direita ou esquerda, não podendo se movimentar nem receber a bola. Outros três novos comandos são adicionados: chutar para esquerda, chutar para direita e chutar para frente, sendo que antigo comando chutar é extinto. Agora, o jogador para fazer o gol deve chutar em um local onde o goleiro não se encontra posicionado. Após a explicação dos novos comandos para os alunos é proposto um novo conjunto de possíveis estratégias, descritas como exercícios, para estimular a fixação das novas regras e novos comandos. Além disso, são propostos exercícios contendo questões com o mesmo objetivo da última aula, porém mais complexos e com o campo inteiro. O objetivo será fazer o gol na goleira superior. A Figura 3 mostra um exemplo de exercício.

\subsection{Tarefa III: Novos Comandos - Toco y Me Voy}

Objetivos: o objetivo desta tarefa é de adicionar um novo conceito ao jogo SoccerCraft - o passe - e quatro novos comandos. O pensamento algorítmico e a análise de custo são 


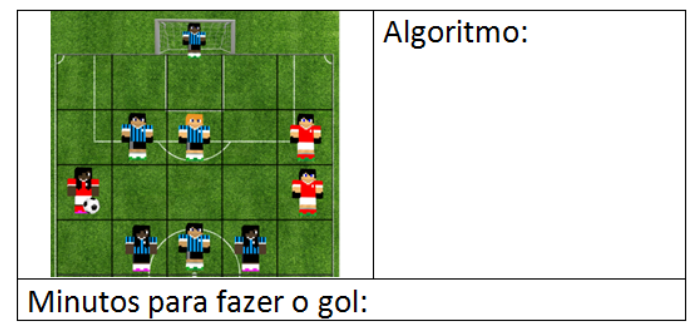

Figura 4. Exemplo de questão utilizada nos exercícios (testes) da aula 3.

explorados nesta tarefa. Material: mesmos materiais da aula anterior (campo, jogadores e bola), jogadores adversários e exercícios utilizando o campo de jogo pela metade ou completo, assim como das primeiras duas aulas, mas com a inclusão de jogadores companheiros. Um exemplo de exercício é mostrado na Figura 4.

Metodologia: a atividade é iniciada relembrando aos alunos como jogar o SoccerCraft e o que foi feito na última aula. Após acontece a introdução a um novo conceito, o passe (ou toque), e quatro novos comandos: tocar para trás, tocar para frente, tocar para esquerda e tocar para direita. Assim, além de jogadores adversários, o jogo também conta com jogadores companheiros. Agora o jogador com a bola também pode tocar a bola para um companheiro de equipe, caso ele esteja em linha reta com o jogador e não haja jogadores adversários no caminho entre eles. Caso haja um jogador companheiro, em linha reta, entre um jogador com a bola e outro jogador companheiro, ao efetuar um passe, o companheiro mais próximo é quem recebe a bola. O jogador que receber a bola é quem deve ser comandado no próximo movimento. O passe tem o mesmo custo dos outros comandos, uma unidade de tempo. Após a explicação dos novos comandos, os alunos devem realizar exercícios contendo questões com o mesmo objetivo da última aula, porém com a possibilidade de utilização dos novos comandos.

\subsection{Tarefa IV: A Peleia}

Objetivos: o objetivo desta aula é oferecer uma tarefa mais descontraída aos alunos, proporcionando um jogo em que potencialmente todas crianças participam, envolvendo o SoccerCraft. O pensamento algorítmico e a análise de custo são explorados nesta tarefa. Material: mesmos materiais da aula anterior (campo, jogadores e bola). Nesta aula não há exercícios ou testes.

Metodologia: neste quarto encontro, acontece uma atividade mais descontraída, um jogo utilizando potencialmente todos os alunos da sala de aula. A turma é inicialmente dividida em duas, sendo cada metade identificando uma equipe no SoccerCraft. Caso o número de jogadores seja ímpar, o professor completa um dos times. Os alunos-jogadores ficam dispostos em dois times, de maneira espelhada pelo campo do jogo. Logo após, deve ser explicado o incremento às regras do jogo à turma, sendo que as regras vindas da aula anterior continuam vigentes: as duas equipes têm uma bola em sua posse e o jogo acontece em turnos, cada equipe tendo direito a um movimento em seu turno. Assim, a equipe que primeiro marcar o gol ganha o jogo. Quando uma equipe chega com a bola dentro da pequena área adversária, acontece um mini jogo. Nesse mini jogo, o goleiro adversário escreve sua estratégia (identificando aonde ele vai se atirar para defender o chute do adversário) em um papel e posteriormente deve escondê-la. Após isso o jogador 


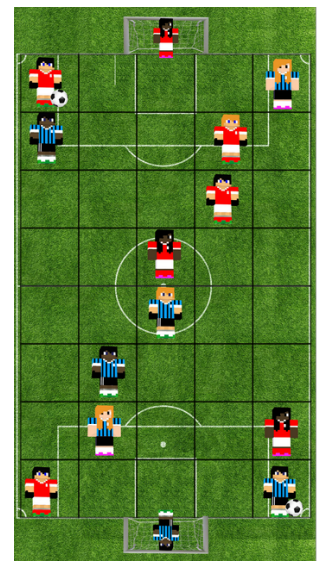

Figura 5. Exemplo de campo de jogo utilizado na aula 4.

com a bola deve escolher o movimento de chute (para esquerda, direita ou frente). Caso ele escolha a estratégia (mesma direção) do goleiro, o gol não é concretizado e a equipe perde o turno, continuando com a bola em posse do jogador dentro da pequena área. Caso contrário, o gol acontece e a partida acaba. A Figura 5 mostra um exemplo de campo espelhado do jogo SoccerCraft de duas equipes de 7 jogadores, envolvendo assim 14 alunos. O número de jogadores no campo pode ser maior que o número de alunos, nesse caso pode-se deixar jogadores menos importantes no campo como não-controláveis ou controláveis somente pelo professor. Caso o número de jogadores seja menor que o número de alunos, alguns alunos devem formar duplas, cada uma controlando um jogador.

O tempo de aula é todo consumido em jogos com os alunos. Vários jogos podem acontecer. O ideal é realizar um rodízio do jogador que começa com a posse de bola, fazendo com que mais alunos participem mais efetivamente dos jogos. Após a realização dos jogos é feita a contagem de quantos jogos cada time ganhou e a divulgação de um vencedor. Um prêmio pode ser dado aos alunos vencedores.

\subsection{Tarefa V: Teste Final}

Objetivos: esta última tarefa tem por finalidade testar os conhecimentos adquiridos pelos alunos com a aplicação de um teste final envolvendo todos os conteúdos trabalhados.

Material: para a realização dessa aula foram utilizados exercícios contendo questões sobre o jogo SoccerCraft além de questões envolvendo conceitos de algoritmos abordados em tarefas anteriores.

Metodologia: propõe-se um teste final contendo seis questões envolvendo habilidades de algoritmos previamente apresentados nas tarefas do jogo, envolvendo ou não o jogo SoccerCraft. A Figura 6 mostra exemplos de questões do teste final.

\section{Validação da Proposta e Resultados Obtidos}

Para validar as estratégias de aprendizagem exploradas no SoccerCraft, várias atividades foram aplicadas em uma turma do sexto ano do Ensino Fundamental da E.M.E.F. Ferreira Vianna para uma amostragem de 19 alunos. A turma era composta por 8 alunos do sexo masculino e 11 alunos do sexo feminino. Em relação ao cronograma, a atividade foi aplicada nos meses de novembro e dezembro de 2017, em cinco encontros. 


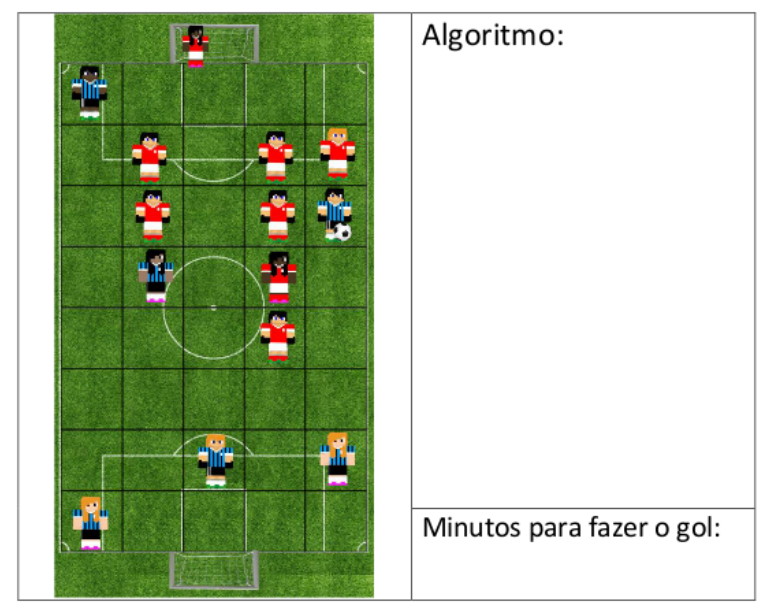

Ajude a galinha a atravessar a rua indicando a ela o menor caminho, escrevendo o algoritmo. Utilize somente os comandos Correr para frente, Correr para esquerda e Correr para direita.

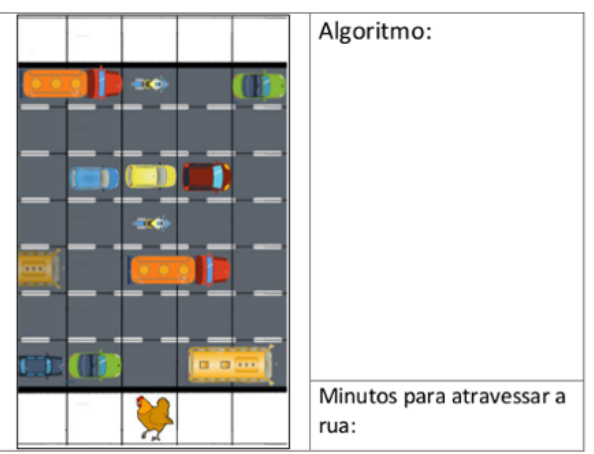

Figura 6. Exemplos de questões do teste final.

Para a realização das atividades foram necessários um responsável por apresentar os conceitos e regras do jogo e até três colaboradores para auxiliar os alunos, quando requisitados. Em todos os encontros havia pelo menos um professor participante do projeto e também o(a) professor(a) da turma, no intuito de auxiliar em características individuais dos alunos.

As avaliações consistiam de um teste escrito com número variado de questões, versando sobre os temas desenvolvidos nas aulas, que totalizavam dez pontos. A descrição do desempenho dos alunos em cada avaliação da atividade é apresentada na Tabela 1. Note-se que o número de alunos avaliados variou de uma tarefa para outra em razão de eventuais ausências nos dias das avaliações.

\begin{tabular}{|c|c|c|c|c|c|c|c|c|c|}
\hline Avaliação & $\mathrm{n}$ & Média & $\mathrm{DP}$ & $\mathrm{CV}(\%)$ & Mínimo & $Q_{1}$ & $\mathrm{Md}$ & $Q_{3}$ & Máximo \\
\hline 1 & 15 & 8 & 3,6 & 44,6 & 0,0 & 6,6 & 10,0 & 10,0 & 10,0 \\
\hline 2 & 18 & 6,7 & 2,9 & 42,9 & 0,0 & 4,7 & 7,5 & 9,0 & 10,0 \\
\hline 3 & 12 & 6,5 & 3,0 & 45,6 & 1,0 & 3,5 & 7,3 & 9,1 & 10,0 \\
\hline 4 & 13 & 6,4 & 2,6 & 40,4 & 1,0 & 5,1 & 6,9 & 8,3 & 9,0 \\
\hline
\end{tabular}

Nota: $\mathrm{n}=$ número de alunos; $\mathrm{DP}=$ desvio padrão; $Q_{1}=$ primeiro quartil; $\mathrm{CV}=$ coeficiente de variação; $\mathrm{Md}=$ mediana; $Q_{3}=$ terceiro quartil.

Nas avaliações da atividade SoccerCraft, segundo a Tabela1, o melhor desempenho foi alcançado na primeira avaliação, na qual a média da turma foi 8,0 e pelo menos metade dos alunos obtiveram nota 10,0. Isso demonstra a facilidade que os alunos tiveram na primeira versão do jogo. Nas três avaliações seguintes a média da turma ficou em torno de 6,5, com pelo menos metade dos alunos atingindo nota igual ou maior que 7,0, evidenciando um bom entendimento dos alunos na atividade proposta, embora tendo piores resultados se comparados à primeira avaliação. Em todas as quatro avaliações a variabilidade das notas foi elevada, com CV variando entre $40 \%$ e $45 \%$. Essa heterogeneidade de notas mostra uma disparidade de entendimento, e/ou ainda de motivação, existente na turma. 


\section{Conclusão}

Este trabalho apresentou a concepção, modelagem e o desenvolvimento de um jogo educacional denominado SoccerCraft, o qual explora conceitos ligados ao futebol utilizando personagens similares aos do jogo Minecraft com o objetivo de desenvolver, via estratégias do PC, a habilidade de pensamento algorítmico a alunos do sexto ano do Ensino Fundamental. Este trabalho também apresentou uma etapa de validação da proposta, na qual os resultados se mostraram bons. Esses resultados mostraram um entendimento dos alunos em relação ao conteúdo proposto, embora tenha havido uma alta discrepância entre algumas notas.

Durante o desenvolvimento das atividades, pôde-se observar um maior engajamento dos alunos nos dois primeiros encontros, aparentemente devido ao SoccerCraft ainda ser uma novidade para eles. Essa motivação diminuiu no terceiro encontro, causando desatenção e consequente dificuldade dos alunos em realizar os exercícios. Já o quarto encontro despertou novamente a motivação das crianças, devido ao espírito de competitividade explorado pela atividade.

Como trabalho futuro, pode-se popor uma continuidade no ensino dos conceitos de algoritmos, com a inserção dos comandos if e while, por exemplo, integrados ao jogo SoccerCraft.

\section{Referências}

Barbosa, D. N. F., Miorelli, S. T., Rasch, L. G., and Silva, C. G. (2017). Ensinando lógica com as tecnologias da informação: Desenvolvendo o raciocínio lógico e o pensamento computacional. CATAVENTOS-Revista de Extensão da Universidade de Cruz Alta, 9:54-72.

Barr, V. and Stephenson, C. (2011). Bringing computational thinking to k-12: what is involved and what is the role of the computer science education community? ACM Inroads, 2(1):48-54.

Batista, E. J. S., Castro Jr, A., Larrea, A. A., and Bogarim, C. A. C. (2015). Utilizando o scratch como ferramenta de apoio para desenvolver o raciocínio lógico das crianças do ensino básico de uma forma multidisciplinar. In Anais do WIE 2015, volume 21, pages 350-359.

Campos, G. et al. (2014). Organização de informações via pensamento computacional: Relato de atividade aplicada no ensino fundamental. In Anais do WIE 2014, pages 390-399.

Carnegie Mellon (2013). Center for Computational Thinking. http://www.cs.cmu.edu/ CompThink/. Acesso: fevereiro/2018.

dos Reis, C. E. R., Duso, G. B., and Webber, C. G. (2018). Robótica educacional aplicada à simulação do sistema digestório. Scientia cum Industria, 5:186-192.

França, R. S., Silva, W. C., and Amaral, C. J. H. (2012). Ensino de ciência da computação na educação básica: Experiências, desafios e possibilidades. In Proc. CSBC'12.

Google (2013). Exploring computational thinking. http://www.google.com/edu/computational-thinking/. Acesso: fevereiro/2018. 
ISTE (2013). Operational definition of computational thinking. http://www.iste.org/learn /computational-thinking/ct-operational-definition. Acesso: fevereiro/2018.

Junior, B., Cavalheiro, S., and Foss, L. (2017). A última árvore: exercitando o pensamento computacional por meio de um jogo educacional baseado em gramática de grafos. In Anais do SBIE 2017, volume 28, page 735.

Kafura, D. and Tatar, D. (2011). Initial experience with a computational thinking course for computer science students. In SIGCSE 2011, pages 251-256. ACM.

Lee, I. et al. (2011). Computational thinking for youth in practice. ACM Inroads, 2(1):3237.

Lugo, M. J. R. and Olabe, X. B. (2018). Diseñando un material educativo digital: nuevas formas de enseñar habilidades del pensamiento computacional. Teknologia berrien erabilera eta gaur egungo Hezkuntza joerak Uso de nuevas tecnologías y tendencias actuales, pages 81-94.

Marques, M., Cavalheiro, S., Foss, L., Avila, C., and Bordini, A. (2017). Uma proposta para o desenvolvimento do pensamento computacional integrado ao ensino de matemática. In Anais do SBIE 2017, volume 28, page 314.

Persson, M. and Bergensten, J. (2011). Minecraft. http://minecraft.net. Acesso: fevereiro/2018.

Santos, E. R. d., Soares, G., Dal Bianco, G., Rocha Filho, J. B. d., and Lahm, R. A. (2016). Estímulo ao pensamento computacional a partir da computação desplugada: uma proposta para educação infantil.

Silva, V., da Silva, L. L., and França, R. (2017). Pensamento computacional na formação de professores: experiências e desafios encontrados no ensino da computação em escolas públicas. In Anais do WIE 2017, volume 23, page 805.

Teixeira, A., Martins, J. R., Batistela, F., Pazinato, A., and Oro, N. (2015). Programação de computadores para alunos do ensino fundamental: A escola de hackers. In Anais do WIE 2017, volume 1, pages 144-163.

TNH1 (2017). Confira os 10 games mais jogados do mundo. http://www.tnh1.com.br/noticias/noticias-detalhe/cultura/confira-os-10-jogos-devideogame-mais-jogados-do-mundo/. Acesso: fevereiro/2018.

UFPel (2018). EXP-PC - Explorando o Pensamento Computacional para a Qualificação do Ensino Fundamental. http://wp.ufpel.edu.br/pensamentocomputacional. Acesso: fevereiro/2018.

Weisshahn, Y., Pinho, G., Cavalheiro, S., Du Bois, A., Aguiar, M., Foss, L., Reiser, R., and de Brum, C. F. (2016). Representação e análise de dados no quinto ano do ensino fundamental: Proposta de atividade e relato de aplicação. In Anais do WIE 2016, volume 22, page 201.

Wing, J. M. (2006). Computational thinking. Communications of the ACM, 49(3):33-35.

Zanetti, H. A. P., Borges, M. A. F., Leal, V. C. G., and Matsuzaki, I. Y. (2017). Proposta de ensino de programação para crianças com scratch e pensamento computacional. Tecnologias, Sociedade e Conhecimento, 4(1):43-58. 